\title{
Carno Completude dos registros de tuberculose no Sistema de Informação de Agravos de Notificação (Sinan) em Santa Catarina, Brasil, 2007-2016*
}

doi: $10.5123 / 51679-49742020000300020$

\section{Completeness of tuberculosis records held on the Notifiable Health Conditions Information System (SINAN) in Santa Catarina, Brazil, 2007-2016}

\section{Completitud de los registros de tuberculosis en el Sistema de Información de Agravamientos de Notificación (Sinan) en Santa Catarina, Brasil, 2007-2016}

\author{
Vanessa Baldez do Canto' ${ }^{-}$(1) orcid.org/0000-0002-6644-0610 \\ Fúlvio Borges Nedel ${ }^{1}$ - (1) orcid.org/0000-0002-8059-7358 \\ ${ }^{1}$ Universidade Federal de Santa Catarina, Centro de Ciências da Saúde, Florianópolis, SC, Brasil
}

\section{Resumo}

Objetivo: descrever a completude dos registros de casos de tuberculose em Santa Catarina, Brasil, no período de 2007 a 2016. Métodos: estudo descritivo, com dados do Sistema de Informação de Agravos de Notificação; para análise de qualidade dos dados, foram consideradas a completude, a consistência dos registros das fichas de notificação e a oportunidade de notificação. Resultados: a completude dos campos obrigatórios da ficha de notificação foi considerada boa; os campos essenciais apresentaram menor completude, destacando-se a 'Escolaridade'; a baixa completude das baciloscopias de acompanhamento e a não atualização dos exames de cultura e sorologia para o HIV demonstram fragilidades no registro do boletim de acompanhamento. Conclusão: os dados do sistema de vigilância da tuberculose foram considerados adequados para orientar as ações de prevenção e controle da doença; recomenda-se o monitoramento e a avaliação periódica dos registros e a adoção de estratégias para melhoria do preenchimento do boletim de acompanhamento.

Palavras-chave: Tuberculose; Vigilância em Saúde Pública; Epidemiologia; Notificação; Sistemas de Informação.

*Artigo originado da dissertação intitulada 'Diabetes mellitus como agravo associado nos casos de tuberculose em Santa Catarina no período entre 2007 e 2016', defendida por Vanessa Baldez do Canto junto ao Programa de Pós-Graduação em Saúde Coletiva da Universidade Federal de Santa Catarina (UFSC) em 2019.

Endereço para correspondência:

Vanessa Baldez do Canto - Rua Desembargador Pedro Silva, n² 2202, Bloco 25, Apto. 23, Coqueiros, Florianópolis, SC, Brasil. CEP: 88080-901

E-mail:baldez.canto@gmail.com 


\section{Introdução}

A tuberculose (TB), apesar de ser uma das doenças mais antigas da humanidade, segue sendo considerada um grave problema de Saúde Pública no mundo, sendo a doença infecciosa que mais leva a óbitos. 0 Brasil se encontra entre os 30 países com alta carga de tuberculose, englobados nesta classificação os dados de incidência, mortalidade e resistência ao tratamento. ${ }^{1}$ Em 2018, a taxa de incidência de TB no país era de 34,8 casos/100 mil habitantes, depois de apresentar uma redução média anual de 1,0\% no período de 2009 a 2018. ${ }^{2}$ Estes resultados, entretanto, são insuficientes para atender às metas da Estratégia pelo Fim da Tuberculose, agenda aprovada pela Assembleia Mundial da Saúde em 2014, quando foi definido o objetivo de eliminar a TB como problema de Saúde Pública do mundo, pautado pelas seguintes metas: (i) a incidência de tuberculose menor que 10/100 mil hab. em 2035, mesma data-limite para (ii) a redução dos óbitos em 95\%, com relação aos óbitos pela doença em $2015 .{ }^{3}$

\section{O Brasil se encontra entre os 30 países com alta carga de tuberculose, englobados nesta classificação os dados de incidência, mortalidade e resistência ao tratamento.}

A taxa de incidência de TB varia muito entre os estados brasileiros. Em 2018, Santa Catarina apresentou 23,7 casos/100 mil hab., taxa menor que a nacional. ${ }^{3}$ Os estados vizinhos, Paraná e Rio Grande do Sul, apresentaram taxas de 19,7 e 40 casos/100 mil hab. respectivamente. ${ }^{2}$ Ao contrário da tendência de decréscimo observada no país, a taxa do estado manteve-se estável no período de 2005 a $2015 .{ }^{4}$

Para avançar rumo ao alcance das metas propostas, é essencial o planejamento de ações a partir do conhecimento da situação epidemiológica da doença. Nesse sentido, a vigilância epidemiológica da TB deve trazer recomendações e fornecer subsídios para a tomada de decisão, sendo suas atribuições (i) a investigação de casos suspeitos, (ii) a notificação de casos confirmados e (iii) a produção de dados do tratamento e acompanhamento dos casos confirmados até o encerramento do tratamento. ${ }^{5}$

A tuberculose é uma doença de notificação compulsória no Brasil desde $1998 .{ }^{6}$ Os dados de notificação são coletados nos estabelecimentos de saúde, a partir das fichas de notificação e do boletim mensal de acompanhamento do paciente, e processados pelo Sistema de Informação de Agravos de Notificação (Sinan). ${ }^{7}$

Estudos apontaram desafios importantes para a vigilância em TB, como a baixa investigação de contatos, ${ }^{8}$ elevada proporção de abandono do tratamento ${ }^{3}$ baixa completude dos campos da fichade notificação e precariedade das informações do diagnóstico, ${ }^{8-10}$ acompanhamento e encerramento dos casos. ${ }^{8-13}$

A baixa qualidade da informação, além de comprometer o conhecimento adequado do perfil epidemiológico dos casos, impede a avaliação das ações de vigilância, visto não ser possível identificar se o não preenchimento decorre de deficiência nas ações da vigilância ou de seu registro. ${ }^{14}$

As pesquisas que avaliam os bancos de dados de TB do Sinan enfocam regiões específicas do país. ${ }^{9-12} 0 \mathrm{~s}$ boletins epidemiológicos, elaborados anualmente pelo Ministério da Saúde e Secretarias de Estado da Saúde, não abordam informações sobre a completude dos dados da ficha, tampouco análises de inconsistência.

Estudos realizados em Santa Catarina, sobre dados do Sinan, não avaliaram a qualidade dos registros. ${ }^{15,16}$ Não foram encontrados trabalhos no estado que analisassem a qualidade do registro dos casos de TB quanto ao preenchimento dos campos da ficha e do boletim de acompanhamento. Estudo nacional com dados municipais, realizado no período de 2001 a 2003, com o propósito de avaliar a qualidade da vigilância epidemiológica, incluindo a completude de cinco campos da ficha de notificação, mostrou Santa Catarina com a melhor situação da região Sul do Brasil. ${ }^{14}$

Diante do exposto, destaca-se a importância da análise da qualidade do banco de dados de TB do Sinan no estado, para identificar sua situação quanto às ações de vigilância. Este estudo teve o objetivo de descrever a completude dos registros de casos de TB em Santa Catarina, no período de 2007 a 2016.

\section{Métodos}

Trata-se de um estudo descritivo, com dados dos casos de TB registrados na base de dados do Sinan de Santa Catarina, no período de 2007 a 2016.

0 estado de Santa Catarina, localizado no Sul do Brasil, constitui-se de 295 municípios desde a outorga da autonomia dos municípios de Balneário Rincão e Pescaria Brava, no ano de 2013. A população do estado, estimada para 0 ano de 2019, é de 7.164 .788 habitantes. ${ }^{17}$ 
A população estudada foi composta por casos de TB com idade a partir de 18 anos, residentes no estado de Santa Catarina, notificados no Sinan, com data de diagnóstico circunscrita ao período de $1^{\circ}$ de janeiro de 2007 a 31 dezembro de 2016. Os critérios do estudo para exclusão de casos foram: ter menos de 18 anos; não residir no estado de Santa Catarina; e apresentar mudança de diagnóstico no decorrer da investigação do caso.

$\mathrm{Na}$ análise de qualidade dos dados, foram consideradas a completude, a consistência dos registros e a oportunidade de notificação. Entende-se por completude a proporção com que cada registro de um sistema de informações apresenta valores não nulos. Os campos considerados nulos ou incompletos são tanto os preenchidos como 'Ignorado' como os deixados em branco. ${ }^{18}$ Para análise da completude dos campos, foi utilizada a classificação proposta pelo Ministério da Saúde: completude boa, quando igual ou superior a 75,1\%; regular, de 75,0 a 50,1\%; baixa de 50,0 a 25,1\%; e muito baixa, quando igual ou inferior a $25,0 \%{ }^{5} \mathrm{~A}$ oportunidade de notificação indica a velocidade de obtenção da informação para ação, ${ }^{19} \mathrm{e}$ no presente estudo, ela foi avaliada pela diferença - em dias - da data do diagnóstico à data da notificação.

Entre os campos existentes na ficha, foram selecionados aqueles cujas variáveis são consideradas estratégicas para o Programa Nacional de Controle da Tuberculose (PNCT) : ${ }^{5}$

a) Sexo

b) Idade

c) Tipo de Entrada

d) Raça/Cor (da pele)

e) Escolaridade

f) Forma (clínica da TB)

g) HIV (buman immunodeficiency virus, ou vírus da imunodeficiência humana)

h) Doenças e Agravos Associados (síndrome da imunodeficiência adquirida [aids], alcoolismo e diabetes)

i) Baciloscopia de Escarro (diagnóstico)

j) Baciloscopias de acompanhamento (escarro) realizadas no $2^{\circ}, 4^{0}$ e $6^{\circ}$ meses de tratamento

k) Situação de Encerramento

Para a análise das baciloscopias de acompanhamento, foram excluídos os casos com a forma clínica extrapulmonar, pois o exame de escarro só é realizado em casos de TB pulmonar. Os conceitos e a forma de preenchimento da ficha de notificação são referidos no
Manual de Recomendações para o Controle de Tuberculose no Brasil e no Dicionário de Dados - Sinan NET., ${ }^{5,20}$

A análise da completude dos campos essenciais foi estratificada por regiões de saúde do estado. As regiões de saúde abordadas pelo estudo encontram-se definidas conforme o Plano Diretor de Regionalização vigente. ${ }^{21}$

Os registros foram extraídos do banco do Sinan de Santa Catarina em 5 de março de 2018, cedidos pela Secretaria de Estado da Saúde após assinatura de Termo de Responsabilidade.

No período de estudo, duas fichas de notificação de TB estiveram vigentes. Na versão da ficha adotada a partir de 2014, alguns campos foram inseridos, excluídos ou alterados, como (i) Uso de Drogas Ilícitas, (ii) Tabagismo, (ii) Beneficiário de programa de transferência de renda do governo, (iii) Terapia Antirretroviral Durante o Tratamento para a TB e (iv) Tratamento Diretamente Observado (TD0). Dadas essas alterações, esses campos não foram analisados.

A notificação pós-óbito consiste no caso de tuberculose que nunca foi registrado no Sinan e foi descoberto após a morte, em decorrência da realização de investigação epidemiológica. Essa categoria foi incluída no campo 'Tipo de Entrada' na ficha vigente a partir de 2014.

Para análise de consistência, foram consideradas duas situações de preenchimento inconsistente dos campos referentes: (i) agravo associado a aids (paciente sabidamente portador de aids) e diagnóstico de HIV negativo; e (ii) resultado positivo de primeira baciloscopia e forma extrapulmonar exclusiva.

A verificação de registros duplicados baseou-se na análise de duplicata, focada nos seguintes campos: Nome do Paciente (criptografado pelo método soundex); Data de Início do Tratamento Atual; Sexo; Tipo de Entrada; Data da Notificação; e Data do Diagnóstico.

Para conferência da atualização dos casos notificados, foi calculado o percentual do preenchimento como 'Em Andamento' nas variáveis 'Cultura de escarro' e 'HIV', nos casos encerrados como 'Cura'. 0 quantitativo de 'Situação de Encerramento' não preenchida indica 0 não fechamento dos casos em tempo oportuno, pois no banco somente constam registros diagnosticados até dezembro de 2016. Considerando-se que o banco foi extraído em março de 2018, contou-se com 15 meses completos a partir da última data de diagnóstico. 0 prazo para encerramento da investigação dos casos de TB é de até 90 dias após o prazo preconizado para conclusão do tratamento. ${ }^{7}$ 
Para as análises de qualidade do banco, foram empregadas análises descritivas. Todas as variáveis foram analisadas quanto a sua completude para os anos de 2007 a 2016. Para análise de tendência do preenchimento dos campos essenciais, foi empregada regressão linear simples. Para verificar a autocorrelação das observações, foi utilizado o teste de Durbin-Watson (DW). Admitiu-se tendência linear estatisticamente significativa quando o coeficiente de regressão atingiu valor $\mathrm{p}<0,05$ e 0 DW um $\mathrm{p}>0,05$.

As análises foram realizadas com auxílio do programa estatístico Stata (Stata Corp College Station, Estados Unidos), versão 14.0. 0 estudo foi aprovado pelo Comitê de Ética em Pesquisa com Seres Humanos da Universidade Federal de Santa Catarina (CEPSH/ UFSC): Parecer $n^{0} 78648417.1 .0000 .0121$, de 21 de novembro de 2017.

\section{Resultados}

De 2007 a 2016, foram diagnosticados 22.741 casos de TB em Santa Catarina. Desses casos, foram excluídos da população do estudo os menores de 18 anos (999), os não residentes no estado (46) e os que apresentaram mudança de diagnóstico no decorrer da investigação do caso (820), restando 20.876 casos a serem analisados. Foram contabilizados 16.640 casos novos, 1.576 recidivas, 1.287 reingressos após abandono, 1.332 transferências, 34 casos sem informação sobre 0 tipo de entrada e 7 notificações pós-óbito.

Não há no banco qualquer registro duplicado. Não foram encontradas inconsistências relacionadas ao campo 'HIV'. Quanto à forma clínica, 55 casos (0,3\%) apresentavam baciloscopia de diagnóstico positiva e forma extrapulmonar exclusiva, caracterizando inconsistência no campo 'Forma'.

$\mathrm{Na}$ análise de completude, encontrou-se $99,9 \%$ de preenchimento nos campos obrigatórios (Sexo; Tipo de Entrada; Forma; Baciloscopia de diagnóstico; Cultura de escarro; HIV).

Os campos essenciais (Raça/Cor [da pele]; Escolaridade; e Agravos Associados) apresentaram menor proporção de preenchimento, com destaque para o campo 'Escolaridade', com 15,2\% de não preenchimento. 0 preenchimento do campo 'Situação de Encerramento' do boletim de acompanhamento foi de $99,1 \%$. Os campos referentes aos agravos associados (alcoolismo; diabetes; aids) apresentaram tendência de crescimento de preenchimento no período; e o campo 'Escolaridade', tendência de decréscimo (Tabela 1).

$\mathrm{Na}$ análise por região de saúde, a Grande Florianópolis e a região denominada Nordeste apresentaram quatro dos cinco campos essenciais analisados com preenchimento menor que $91 \%$ (Tabela 2). Na análise de não preenchidos no campo 'Raça/Cor', a região da Grande Florianópolis foi responsável por 45,34\% dos não preenchidos, seguida pela Nordeste, com $36,65 \%$, e pelo Médio Vale do Itajaí, com 6,55\%. No campo 'Escolaridade', a Grande Florianópolis contribuiu com 36,77\% dos não preenchidos, seguida da Nordeste, com 24,95\%, e da Foz do Rio Itajaí, com 9,07\%. No campo 'Diabetes', 49,92\% dos não preenchidos foram da região da Grande Florianópolis, seguida da Nordeste, com 31,23\%, e da Foz do Rio Itajaí, com 8,45\%. No campo 'Alcoolismo', a Grande Florianópolis foi responsável por 44,57\% dos não preenchidos, seguida pela Nordeste, com 30,11\%, e pela Foz do Rio Itajaí com 6,31\%. No campo 'Aids', a Grande Florianópolis respondeu por $41,10 \%$ dos não preenchidos, seguida da Nordeste, com 15,51\%, e da Foz do Rio Itajá, com 14,34\% (Tabela 2).

No período analisado, $6,12 \%$ dos registros apresentaram cultura de escarro 'Em Andamento' e 2,13\% do campo 'HIV' com 0 mesmo preenchimento. 0 preenchimento do boletim de acompanhamento permite a atualização das informações referentes a esses campos preenchidas como 'Em Andamento' no momento da notificação. Nos casos encerrados pelo boletim como 'Cura', 1,84\% ainda mantinha o resultado do HIV como 'Em Andamento' e 4,83\% do exame de cultura de escarro na mesma situação (Tabela 3).

As baciloscopias de acompanhamento apresentaram menor completude que as de diagnóstico e diminuíram 0 percentual de preenchimento no decorrer dos meses de acompanhamento, chegando a $29,5 \%$ de não preenchimento no $6^{\circ}$ mês de tratamento. Considerando-se apenas os casos com forma clínica pulmonar, as baciloscopias de acompanhamento ainda apresentaram campos preenchidos com 'Não se aplica' e 'Não Realizado', quando o código 'Não se aplica' se destina tão somente aos casos de apresentação extrapulmonar (Tabela 4).

No período estudado, as notificações foram realizadas em até 7 dias após a data do diagnóstico em 69,5\% dos casos. 0 número mediano de dias de atraso da notificação foi 0 , demonstrando que metade dos casos foram notificados no mesmo dia $(50,2 \%)$. A variabilidade, entretanto, é grande, com intervalo interquartílico de 


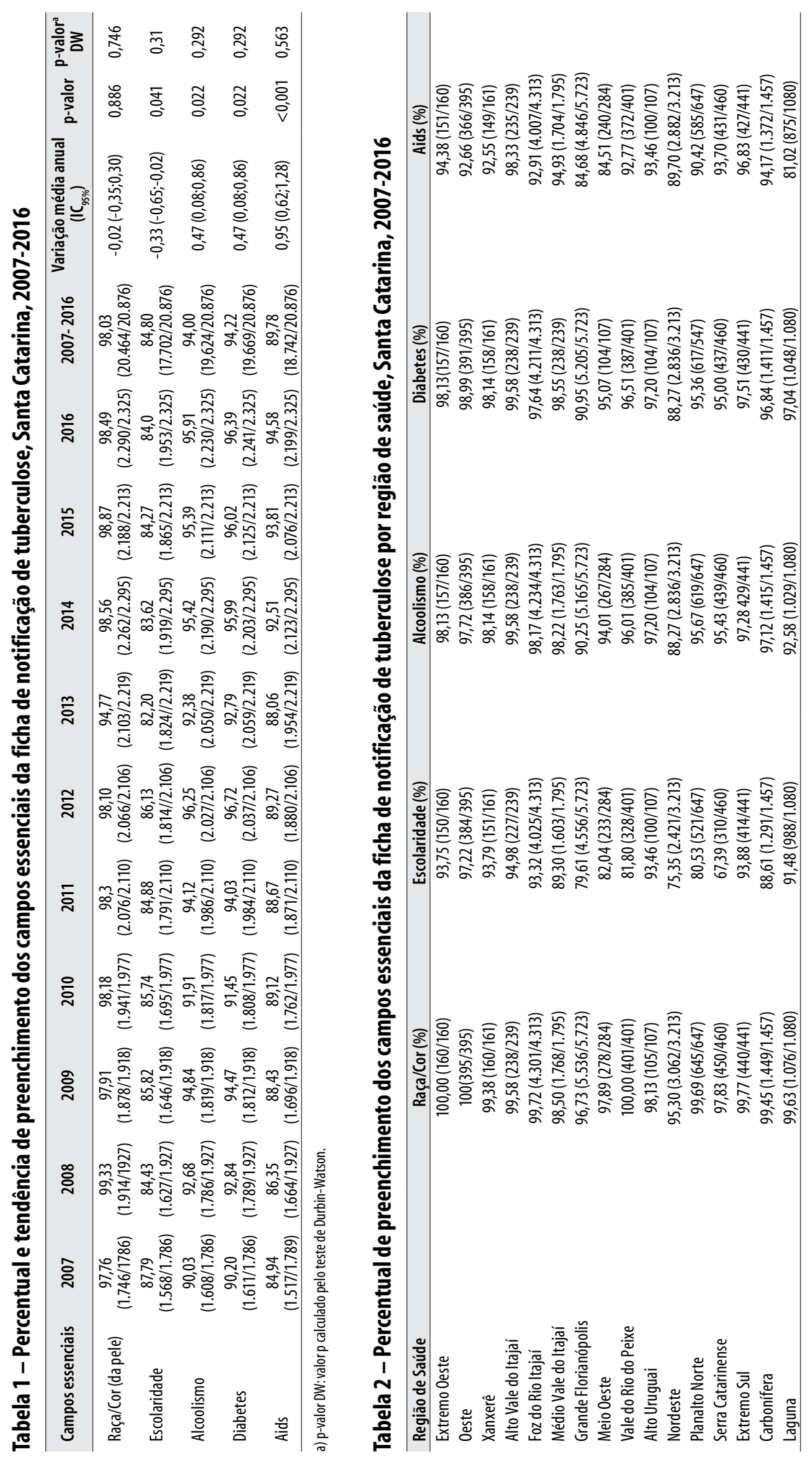


Tabela 3 - Preenchimento dos campos de exames HIVa e Cultura, na ficha de notificação e no boletim de acompanhamento de tuberculose do Sinan, ${ }^{\mathrm{b}}$ Santa Catarina, 2007-2016

\begin{tabular}{lccccc}
\hline Variável & Positivo (\%) & Negativo (\%) & Em andamento (\%) & Não realizado (\%) & Não preenchido (\%) \\
\hline Ficha de notificação & & & & & \\
\hline HIV $^{\mathrm{a}}$ & $23,90(4.989 / 20.876)$ & $62,10(12.963 / 20.876)$ & $2,13(444 / 20.876)$ & $11,87(2.477 / 20.876)$ & $0,01(3 / 20.876)$ \\
$\quad$ Cultura de escarro & $31,35(6.544 / 20.876)$ & $18,15(3.790 / 20.876)$ & $6,12(1.277 / 20.876)$ & $44,37(9.262 / 20.876)$ & $0,01(3 / 20.876)$ \\
\hline Boletim de acompanhamento & & & & \\
\hline HIV $^{\mathrm{a}}$ & $17,48(2.547 / 14.569)$ & $70,14(10.218 / 14.569)$ & $1,84(268 / 14.569)$ & $10,54(1.536 / 14.569)$ & - \\
Cultura de escarro & $32,47(4.730 / 14.569)$ & $20,52(2.989 / 14.569)$ & $4,83(704 / 14.569)$ & $42,19(6.146 / 14.569)$ & - \\
\hline
\end{tabular}

a) HIV: human immunodeficiency virus (vírus da imunodeficiência humana).

b) Sinan: Sistema de Informação de Agravos de Notificação.

c) Casos encerrados como 'Cura'

\section{Tabela 4 - Preenchimento dos campos de baciloscopia de diagnóstico e baciloscopia de acompanhamento

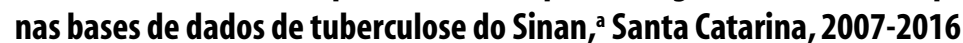

\begin{tabular}{|c|c|c|c|c|c|}
\hline Variável & Positivo (\%) & Negativo (\%) & Não se aplica (\%) & Não realizado (\%) & Não preenchido (\%) \\
\hline Baciloscopia - diagnóstico & $49,95(10.427 / 20.876)$ & $31,48(6.571 / 20.876)$ & $0,58(121 / 20.876)$ & $17,98(3.754 / 20.876)$ & $0,01(3 / 20.876)$ \\
\hline Baciloscopia no $1^{\circ}$ mês & $31,59(5.576 / 17.651)$ & $28,99(5.117 / 17.651)$ & $0,21(37 / 17.651)$ & $23,50(4.148 / 17.651)$ & $15,71(2.773 / 17.651)$ \\
\hline Baciloscopia no $2^{\circ}$ mês & $10,23(1.805 / 17.651)$ & $36,05(6.364 / 17.651)$ & $0,21(37 / 17.651)$ & $35,55(6.275 / 17.651)$ & $17,96(3.170 / 17.651)$ \\
\hline Baciloscopia no $3^{\circ}$ mês & 4,51 (796/17.651) & $35,68(6.298 / 17.651)$ & $0,21(37 / 17.651)$ & 37,64 (6.643/17.651) & $21,96(3.877 / 17.651)$ \\
\hline Baciloscopia no $4^{\circ}$ mês & 2,48 (437/17.651) & $36,68(6.475 / 17.651)$ & $0,21(37 / 17.651)$ & $37,03(6.537 / 17.651)$ & $23,60(4.165 / 17.651)$ \\
\hline Baciloscopia no $5^{\circ}$ mês & $1,54(272 / 17.651)$ & $34,20(6.037 / 17.651)$ & $0,21(37 / 17.651)$ & $36,68(6.474 / 17.651)$ & $27,37(4.831 / 17.651)$ \\
\hline Baciloscopia no $6^{\circ}$ mês & $1,12(197 / 17.651)$ & $39,75(7.016 / 17.651)$ & $0,21(37 / 17.651)$ & $29,44(5.197 / 17.651)$ & $29,48(5.204 / 17.651)$ \\
\hline
\end{tabular}

a) Sinan:Sistema de Informação de Agravos de Notificação.

14 dias $(\mathrm{Q} 1=0 ; \mathrm{Q3}=14)$. Ao se analisar a oportunidade da notificação pelo tipo de entrada, o percentual de casos notificados em até 7 dias foi de $74,6 \%$ nos casos novos e de $49,5 \%$ nos demais tipos de entrada. Sete municípios $(2,4 \%)$ não tiveram caso registrado no período. Em $88,4 \%$ dos casos, a notificação foi feita no município de residência do indivíduo.

\section{Discussão}

A completude dos campos analisados da ficha de notificação da tuberculose em Santa Catarina foi considerada boa, segundo os parâmetros do Ministério da Saúde. Os campos classificados como essenciais apresentaram menor completude, quando comparados aos campos de preenchimento obrigatório. A menor completude das baciloscopias de acompanhamento e a não atualização dos campos preenchidos como 'Em Andamento' no momento da notificação podem indicar falhas no uso do boletim de acompanhamento dos casos de tuberculose. Estudo realizado com dados do Sinan relativos ao período de 2001 a 2006 corrobora esse achado, ao encontrar preenchimento insatisfatório $(<80 \%)$ das variáveis referentes ao acompanhamento em Fortaleza, Salvador e Rio de Janeiro. ${ }^{13}$
A consistência do sistema de informação, avaliada neste estudo pelos campos 'HIV' e 'Forma', foi excelente. Entretanto, cabe destacar que, a partir do preenchimento do campo 'Aids' como 'Sim', o Sinan automaticamente preenche o campo 'HIV' como Positivo.

Problemas quanto ao preenchimento das variáveis sociodemográficas também foram encontrados em outros estudos. ${ }^{10,13}$ Considerando-se que condições socioeconômicas, a exemplo da baixa escolaridade, estão associadas a piores desfechos em TB, como abandono, falência de tratamento e óbito, ${ }^{22} 0$ conhecimento sobre condições socioeconômicas dos casos é imprescindível à análise da população afetada.

A análise de informação válida (onde se consideram além dos campos em branco e ignorados, os campos preenchidos como 'Em andamento' para a análise de completude) pode sugerir a não investigação dessas questões ou a falta de atualização da informação no boletim de acompanhamento do caso. No período analisado, mais de $50 \%$ das fichas apresentaram o campo 'Cultura' preenchido como 'Em Andamento' ou 'Não Realizado'. A cultura é um método de elevada especificidade e sensibilidade no diagnóstico da TB. Ela é indicada, independentemente do resultado da baciloscopia, nos casos de pacientes com antecedentes 
de tratamento prévio, imunodeprimidos ou com baciloscopia positiva no final do $2^{\circ}$ mês de tratamento. ${ }^{5}$

O campo 'HIV' apresentou $14,5 \%$ dos registros como 'Não Realizado' ou 'Em Andamento'. Vale ressaltar que a partir de 2014, com a nova versão do Sinan, casos que apresentaram 'HIV' e 'Cultura' preenchidos como 'Em Andamento' são alterados automaticamente, no encerramento, para 'Não Realizado' ${ }^{20} \mathrm{~A}$ partir de um estudo realizado na Bahia, o percentual do campo 'HIV' preenchido com esses códigos somou mais de $57 \%{ }^{11}$ Sanchez et al. relataram que a sorologia para HIV estava indisponível em 32\% dos casos de TB notificados no Brasil, entre 2003 e $2008,{ }^{23}$ embora, desde 0 ano de 1998, seja recomendada a oferta de teste de HIV a todos os casos de TB. ${ }^{24}$

A diferença encontrada entre a completude dos campos 'HIV' e 'Aids' pode estar relacionada às diretrizes para preenchimento das fichas: preencher o campo 'HIV' é obrigatório para registro da notificação no Sinan. ${ }^{7}$ Recomenda-se a revisão dos documentos técnicos referentes à classificação das variáveis obrigatórias no Sinan, como uma estratégia visando aumentar a completude dos campos não obrigatórios. Dos 4.989 casos positivos para 0 HIV, 90,1\% apresentavam aids como agravo associado, o que indica não tratamento do HIV levando a aids e, por conseguinte, menor imunidade..$^{20}$ Neste contexto, os programas de controle de TB e de HIV/aids das três esferas de gestão devem implantar estratégias para expandir os cuidados em saúde nessa população, visto que o risco de desfecho desfavorável (abandono, recidiva, óbito) é aproximadamente três vezes maior nos pacientes com coinfecção TB/aids. ${ }^{23,24}$

Observou-se qualidade boa de completude do campo de baciloscopia de diagnóstico. Entretanto, ao se analisar a completude das baciloscopias de acompanhamento do boletim, o percentual de preenchimento diminuiu.

Estudo realizado nos centros urbanos com maior carga de TB no país identificou que, de 2001 a 2006, a baciloscopia nos $2^{\circ}, 4^{0}$ e $6^{\circ}$ meses encontrava-se entre as variáveis com menor proporção de preenchimento. Seus autores sugerem que o fato pode indicar, além de falha no preenchimento das fichas, falta de acompanhamento dos casos de TB pelas equipes de saúde..$^{13} 0$ Ministério da Saúde recomenda a realização mensal de baciloscopia nos casos de TB pulmonar, sendo indispensáveis as do $2^{\circ}, 4^{0}$ e $6^{0}$ meses. A considerar a hipótese levantada pelo estudo mencionado, recomenda-se a realização de estudos transversais que analisem 0 acompanhamento dos casos, assim como o registro das informações referentes ao acompanhamento.

A oportunidade do sistema de vigilância revelou boa agilidade em obter informações, notificando 69,5\% dos casos em até 7 dias após o diagnóstico, resultado acorde com o preconizado pelo Ministério da Saúde. ${ }^{18} \mathrm{Em}$ $50,2 \%$ dos casos, a notificação foi realizada no mesmo dia do diagnóstico. Estudo realizado no Brasil, sobre o período de 2005 a 2008 , revelou que cerca de $55 \%$ dos casos de TB foram notificados no mesmo dia do diagnóstico, indicando que o sistema de vigilância da doença no país é oportuno na notificação dos casos. ${ }^{25}$

0 não preenchimento de determinados campos pode estar relacionado à percepção de alguns profissionais da Saúde de que as fichas de notificação representam uma questão meramente burocrática, desconsiderando a produção de dados e informações como ações necessárias à gerência das ações e serviços. ${ }^{9}$ Além disso, pesquisa realizada em cinco municípios do país, no ano de 2011, encontrou deficiências na capacitação dos profissionais da vigilância da TB. ${ }^{26}$ Diante disso, sugere-se a realização de estudos capazes de identificar lacunas de conhecimento e competência dos profissionais quanto aos registros de vigilância, visando subsidiar as ações necessárias para a melhoria das notificações. A mudança dessa realidade possibilita aos programas reforçarem, em suas rotinas, práticas adequadas às recomendadas pelo PNCT, como linkage, e análise de completude e de consistência dos dados. ${ }^{8}$

Mais de $10 \%$ dos casos foram notificados em municípios diferentes do município de residência. Para a melhoria do sistema de vigilância da TB, tanto na detecção de casos como em seu acompanhamento, faz-se necessário o fortalecimento da Atenção Básica e a efetivação de suas responsabilidades no controle da TB, especialmente a identificação de sintomáticos respiratórios, busca ativa de faltosos, tratamento e acompanhamento dos casos. O Ministério da Saúde aponta a Atenção Básica do Sistema Único de Saúde (SUS), particularmente a Estratégia Saúde da Família (ESF), como a principal porta de entrada para o sintomático respiratório e para a pessoa com TB no sistema de Saúde Pública. Os atributos da Atenção Primária à Saúde promovem maior vinculação entre pacientes e profissionais de saúde e assim, se favorece a realização de atividades como a busca ativa, investigação dos contatos, tratamento diretamente observado (TD0) e acompanhamento do caso. ${ }^{27} 0$ aumento da cobertura 
das equipes de ESF em Curitiba no período de 2000 e 2009, por exemplo, esteve associado à redução da proporção de abandono do tratamento da TB. ${ }^{28}$

Recomenda-se o estabelecimento de metas para o Plano Estadual de Enfrentamento da TB, em nome do aprimoramento da qualidade do registro e da retomada de indicadores da TB nas pactuações do SUS, com vistas a revisar o financiamento do sistema de vigilância da doença. No período de estudo, sete municípios catarinenses (São João do Oeste, Palma Sola, Sul Brasil, Águas de Chapecó, Cunhataí, Urupema e Marema) não apresentaram qualquer caso de TB entre seus residentes. Trata-se de municípios com menos de 10 mil hab. - quatro deles, menos de 3 mil hab. -, situados em regiões de altas taxas de TB. Importante salientar que municípios sem casos, localizados em áreas de alto risco para a ocorrência de TB, precisam ser analisados com cautela, haja vista a possibilidade de subnotificação. ${ }^{29}$ Em casos como esses, recomenda-se 0 monitoramento da proporção de sintomáticos respiratórios e de contatos examinados, para avaliação da qualidade do sistema de vigilância municipal na deteç̧ão de casos. A realização de estudos de inquérito populacional e nos serviços de saúde, para identificação de sintomáticos respiratórios, pode auxiliar na análise de falhas na detecção de casos.

A verificação periódica da completude dos dados também deve fazer parte das ações de vigilância no município, assim como a análise de inconsistência e duplicatas, conforme recomenda o Ministério da Saúde. ${ }^{5}$

Os resultados do presente estudo permitiram traçar um panorama do registro de notificação de TB em Santa Catarina. A região da Grande Florianópolis

\section{Referências}

1. World Health Organization. Global tuberculosis report 2017 [Internet]. Geneva: World Health Organization; 2017 [cited 2020 May 22]. 147 p. Available from: https://www.who.int/tb/publications/global_report/ gtbr2017_main_text.pdf?ua=1

2. Ministério da Saúde (BR). Secretaria de Vigilância em Saúde. Departamento de Vigilância Epidemiológica. Brasil livre da tuberculose: evolução dos cenários foi a que mais contribuiu para o não preenchimento dos campos essenciais, mostrando ser necessário um olhar específico sobre 0 registro das notificações nessa região. Os dados do sistema de vigilância da TB foram, de maneira geral, adequados para orientar as ações de prevenção e controle da doença. Entretanto, eles podem ser melhorados. No período avaliado, os campos referentes aos agravos associados (alcoolismo, diabetes e aids) apresentaram aumento no preenchimento, enquanto o campo 'Escolaridade' apresentou decréscimo, e o preenchimento do campo 'Raça/Cor' (da pele) permaneceu estável. Foram demonstradas fragilidades nos registros da notificação e do boletim de acompanhamento.

0 presente estudo traz informação original para a vigilância de TB no estado, servindo de subsídio aos gestores e trabalhadores de saúde, tanto do estado de Santa Catarina como de seus municípios, para a tomada de decisões, conjuntas buscando melhorar os processos de trabalho das equipes de saúde e qualificar as informações referentes à tuberculose, contribuindo assim para 0 controle da doença.

\section{Contribuição dos autores}

Canto VB e Nedel FB contribuíram com a concepção e delineamento do estudo, análise e interpretação dos dados e redação da primeira versão do manuscrito. Nedel FB contribuiu, significativamente, com a revisão crítica do conteúdo intelectual do manuscrito. Ambos os autores aprovaram a versão final e são responsáveis por todos os aspectos do trabalho, incluindo a garantia de sua precisão e integridade. epidemiológicos e operacionais da doença. Bol Epidemiol [Internet]. 2019 mar [citado 2020 maio 22];50(9):1-18. Disponível em: https://www.saude.gov. br/images/pdf/2019/marco/22/2019-009.pdf

3. World Health Organization. The end TB strategy [Internet]. Geneva; World Health Organization; 2015 [cited 2020 May 22]. Available from: https://www.who. int/tb/post2015_strategy/en/ 
4. Secretaria de Estado de Saúde (Santa Catarina). Situação da tuberculose em Santa Catarina, 2001 2015. Inf Epidemiol Barriga Verde [Internet]. 2017 mar [citado 2020 maio 22];14(esp.):1-4. Disponível em: http://dive.sc.gov.br/conteudos/publicacoes/ Informativo_Tuberculose-2017.pdf

5. Ministério da Saúde (BR). Secretaria de Vigilância em Saúde. Departamento de Vigilância Epidemiológica. Manual de recomendações para o controle da tuberculose no Brasil [Internet]. 2. ed. Brasília: Ministério da Saúde; 2019 [citado 2020 maio 22]. 364 p. Disponível em: http://dive. sc.gov.br/conteudos/agravos/publicacoes/manualrecomendacoestuberculose-20mar19-isbn.pdf

6. Brasil. Ministério da Saúde. Portaria GM/MS n ${ }^{0}$ 2325, de 08 de dezembro de 2003. Define a relação de doenças de notificação compulsória para todo território nacional [Internet]. Diário Oficial da União, Brasília (DF), 2003 dez 10 [citado 2020 maio 22];Seção 1:81. Disponível em: http:// bvsms.saude.gov.br/bvs/saudelegis/gm/2003/ prt2325_08_12_2003.html

7. Ministério da Saúde (BR). Secretaria de Vigilância em Saúde. Departamento de Vigilância Epidemiológica. Roteiro para análise da base de dados de tuberculose do sistema de informação de agravos de notificação SINAN e cálculo de indicadores básicos [Internet]. Brasília: Ministério da Saúde; 2006 [citado 2020 maio 22]. 52 p. Disponível em: http://www.saude.ba.gov. br/wp-content/uploads/2017/12/Caderno-de-analiseTuberculose.pdf

8. Silva MDG, Bartholomay P, Cruz GO, Garcia PL. Avaliação da qualidade dos dados, oportunidade e aceitabilidade da vigilância da tuberculose nas microrregiões do Brasil. Ciênc Saúde Coletiva [Internet]. 2017 out [citado 2020 jan 26];22(10):3307-19. Disponível em: https://doi. org/10.1590/1413-812320172210.18032017

9. Santos NP, Lírio M, Passos LAR, Dias JP, Kritski AL, Galvão-Castro B, et al. Completude das fichas de notificações de tuberculose em cinco capitais do Brasil com elevada incidência da doença. J Bras Pneumol [Internet]. 2013 abr [citado 2020 jan 26];39(2):221225. Disponível em: https://doi.org/10.1590/S180637132013000200014

10. Moreira CMM, Maciel ELN. Completude dos dados do Programa de Controle da Tuberculose no Sistema de
Informação de Agravos de Notificação no Estado do Espírito Santo, Brasil: uma análise do período de 2001 a 2005. J Bras Pneumol [Internet]. 2008 abr [citado 2020 jan 26];34(4):225-229. Disponível em: https:// doi.org/10.1590/S1806-37132008000400007

11. Lírio M, Santos NP, Passos LAR, Kritski A, Galvão-Castro B, Grassi MFR. Completude das fichas de notificação de Tuberculose nos municípios prioritários da Bahia para controle da doença em indivíduos com HIV/AIDS. Ciênc Saúde Coletiva [Internet]. 2015 abr [citado 2020 jan 26];20(4):1143-1148. Disponível em: https://doi.org/10.1590/1413-81232015204.00672014

12. Medeiros D, Sucupira DE, Guedes RM, Costa AJL. Análise da qualidade das informações sobre tuberculose no município de Belford Roxo, Rio de Janeiro, 2006 a 2008. Cad Saúde Colet [Internet]. 2012 abr [citado 2020 jan 26];20(2):146-52. Disponível em: http://www.cadernos.iesc.ufrj.br/cadernos/images/ csc/2012_2/artigos/csc_v20n2_146-152.pdf

13. Malhão TA, Oliveira GP, Codennoti SB, Moherdaui F. Avaliação da completude do Sistema de Informação de Agravos de Notificação da Tuberculose, Brasil, 20012006. Epidemiol Serv Saúde [Internet]. 2010 jul-set [citado 2020 jan 26];19(3):245-56. Disponível em: http://dx.doi.org/10.5123/S1679-49742010000300007

14. Braga JU. Vigilância epidemiológica e o sistema de informação da tuberculose no Brasil, 2001-2003. Rev Saúde Pública [Internet]. 2007 set [citado 2020 jan 26];41(1):77-87. Disponível em: https://doi. org/10.1590/S0034-89102007000800011

15. Ferrer GCN, Silva RM, Ferrer KT, Traebert J. A carga de doença por tuberculose no estado de Santa Catarina. J Bras Pneumol [Internet]. 2013 jan-fev [citado 2020 jan 26];39(6):61-8. Disponível em: https://doi. org/10.1590/S1806-37132014000100009

16. Mendonça SA, Franco SC. Avaliação do risco epidemiológico e do desempenho dos programas de controle de tuberculose nas Regiões de Saúde do estado de Santa Catarina, 2003 a 2010. Epidemiol Serv Saúde [Internet]. 2015 jan-mar [citado 2020 jan 26];24(1):59-70. Disponível em: https://doi. org/10.5123/S1679-49742015000100007

17. Instituto Brasileiro de Geografia e Estatísticas - IBGE. Portal cidades e estados [Internet]. Rio de Janeiro: Instituto Brasileiro de Geografia e Estatísticas; 2020 [citado 2020 mar 21. Disponível em https://www.ibge. gov.br/cidades-e-estados/sc.html 
18. Ministério da Saúde (BR). Secretaria de Vigilância em Saúde. Departamento de Vigilância Epidemiológica. Manual de recomendações para o controle da tuberculose no Brasil [Internet]. Brasília: Ministério da Saúde; 2011 [citado 2020 maio 22]. 284 p. Disponível em: http://bvsms.saude.gov.br/bvs/ publicacoes/manual_recomendacoes_controle_ tuberculose_brasil.pdf

19. World Health Organization. Communicable disease surveillance and response systems: guide to monitoring and evaluating [Internet]. Geneva: World Health Organization; 2006 [cited 2020 May 22]. Available from: https:/www.who.int/csr/resources/ publications/surveillance/WHO_CDS_EPR_ LY0_2006_2/en/

20. Ministério da Saúde (BR). Secretaria de Vigilância em Saúde. Departamento de Vigilância Epidemiológica. Dicionário de dados - SINAN NET - Versão 5.0: tuberculose [Internet]. Brasília: Ministério da Saúde; 2019 [citado 2020 mar 21]. Disponível em: http:// portalsinan.saude.gov.br/tuberculose

21. Secretaria de Estado da Saúde (Santa Catarina). Plano diretor de regionalização: PDR 2008 [Internet]. Florianópolis: Secretaria de Estado da Saúde; 2008 [citado 2013 out 27]. 136 p. Disponível em: http:// www.saude.sc.gov.br/index.php/documentos/ informacoes-gerais/planejamento-em-saude/ instrumentos-de-gestao-estadual/plano-diretorde-regionalizacao/2524-plano-diretor-deregionalizacao-2008/file

22. Albuquerque MFPM, Ximenes RAA, Lucena-Silva N, Souza WV, Dantas AT, Rodrigues LC, et al. Factors associated with treatment failure, dropout, and death in a cohort of tuberculosis patients in Recife, Pernambuco, Brazil. Cad Saúde Pública [Internet]. 2007 Jul [cited 2020 Jan 27];23(7):1573-82. Available from: https://doi.org/10.1590/s0102$311 \times 2007000700008$

23. Sanchez M, Bartholomay P, Arakaki-Sanchez D, Enarson D, Bissell K, Barreira D, et al. Outcomes of TB treatment by HIV status in national recording systems in Brazil, 2003-2008. PLoS One [Internet].
201 Mar [cited 2020 Jan 27];7(3):e33129. Available from: https://doi.org/10.1371/journal.pone.0033129

24. Ministério da Saúde (BR). Secretaria de Vigilância em Saúde. Departamento de Vigilância Epidemiológica. CoinfeçãoTB-HIV no Brasil: panorama epidemiológico e atividades colaborativas 2017. Bol Epidemiol [Internet]. 2017 jun [citado 2020 maio 22]. Disponível em: http://www.aids.gov.br/pt-br/pub/2017/ coinfeccao-tb-hiv-no-brasil-panorama-epidemiologicoe-atividades-colaborativas-2017

25. Mandacarú PMP. Oportunidade do sistema de vigilância da dengue, doenças exantemáticas, meningite e tuberculose no Brasil [dissertação]. Goiânia (G0): Universidade Federal de Goiás; 2012. Disponível em: https://repositorio.bc.ufg.br/tede/ handle/tede/3568

26. Scatena LM, Wysocki AD, Beraldo AA, Magnabosco GT, Brunello MEF, Rufino Netto A, et al. Validação e confiabilidade: instrumento para avaliação de serviços que tratam tuberculose. Rev Saúde Pública [Internet]. 2015 fev [citado 2020 jan 26];49(7):1-11. Disponível em: http://dx.doi.org/10.1590/S00348910.2015049005548

27. Cardozo-Gonzales RI, Palha PF, Harter J, Alarcon E, Lima LM, Tomberg JO. Avaliação das ações de detecção de casos de tuberculose na atenção primária. Rev Eletrônica Enferm [Internet]. 2015 out-dez [citado 2020 jan 26];17(4). Disponível em: https:/doi. org/10.5216/ree.v17i4.32846

28. Marquieviz J, Alves IS, Neves EB, Ulbricht L. A estratégia de saúde da família no controle da tuberculose em Curitiba (PR). Ciên Saúde Coletiva [Internet]. 2013 jan [citado 2020 jan 26];18(1):265-71. Disponível em: http://dx.doi.org/10.1590/S141381232013000100027

29. Oliveira GP, Pinheiro RS, Coeli CM, Barreira D, Codenotti SB. Uso do sistema de informação sobre mortalidade para identificar subnotificação de casos de tuberculose no Brasil. Rev Bras Epidemiol [Internet]. 2012 set [citado 2020 jan 26];15(3):46877. Disponível em: http://dx.doi.org/10.1590/\$1415790X2012000300003 


\section{Abstract}

Objective: to describe the completeness of tuberculosis (TB) case records in Santa Catarina, Brazil, from 2007 to 2016. Methods: this was a descriptive study using Notifiable Health Conditions Information System data; completeness, consistency of notification form records and timely notification were analyzed in order to assess data quality. Results: completeness of notification form mandatory fields was considered good; essential fields were less complete, in particular the 'Schooling' field; low completeness of follow-up sputum smear microscopy and failure to update culture tests and HIV serology tests demonstrate weaknesses in follow-up records. Conclusion: the tuberculosis surveillance system data were considered adequate for guiding tuberculosis prevention and control actions; record monitoring and periodical evaluation, as well as adoption of strategies to improve follow-up report completeness are recommended.

Keywords: Tuberculosis; Public Health Surveillance, Epidemiology; Notification; Information Systems.

\section{Resumen}

Objetivo: describir la completitud de los registros de casos de tuberculosis en Santa Catarina, Brasil, en el periodo de 2007 a 2016. Métodos: estudio descriptivo con datos del Sistema de Información de Agravamientos de Notificación; para análisis de la calidad de los datos fueron consideradas la completitud, la consistencia de los registros de las fichas de notificación y la oportunidad de notificación. Resultados: la completitud de los campos obligatorios de la ficha de notificación fue considerada buena; los campos esenciales presentaron menos completitud, destacándose el campo "Escolaridad"; la baja completitud de las baciloscopias de acompañamiento y la no actualización de los exámenes de cultivo y serología para el VIH demuestran fragilidades en el registro del formulario de seguimiento. Conclusión: los datos del sistema de vigilancia de la tuberculosis fueron considerados adecuados para orientar acciones de prevención y control de la enfermedad; se recomienda el monitoreo y la evaluación periódica de los registros y la adopción de estrategias para mejorar el llenado del formulario de seguimiento.

Palabras clave: Tuberculosis; Vigilancia en Salud Pública, Epidemiología; Notificación; Sistemas de Información.

Recebido em 28/01/2020

Aprovado em 11/05/2020 